had undergone stenting (1.26\%) and those who were treated with endarterectomy (1.97\%).

Although stenting seems to be an effective alternative to endarterectomy for medium-term prevention of ipsilateral stroke, higher periprocedural risk of stroke or death, and recurrent stenosis, are issues for concern.

Original articles Eckstein H-H et al. (2008) Results of the stent-protected angioplasty versus carotid endarterectomy (SPACE) study to treat symptomatic stenoses at 2 years: a multinational, prospective, randomised trial. Lancet Neurol 7: 893-902

Mas J-L et al. (2008) Endarterectomy versus angioplasty in patients with symptomatic severe carotid stenosis (EVA-3S) trial: results up to 4 years from a randomised, multicentre trial. Lancet Neurol 7: 885-892

\section{Drug-eluting stents: biodegradable versus durable polymers}

Very late stent thrombosis, which was associated with first-generation drug-eluting (sirolimus or paclitaxel) stents, has been attributed at least in part to the durable polymer coatings used to control drug release. New research has shown promising results from a second-generation stent, that elutes biolimus - a semisynthetic, highly lipophilic analog of sirolimus - via breakdown of a biodegradable polymer over a period of 6-9 months after implantation.

Windecker et al. compared the novel biolimuseluting stent with an established, FDA approved, sirolimus-eluting stent in the management of patients undergoing percutaneous coronary intervention. The European, multicenter, noninferiority trial included patients with at least one lesion suitable for coronary stent implantation. Patients received at least one novel (847 patients, 1,226 lesions) or conventional (823 patients, 1,163 lesions) stent.

The primary clinical end point-a composite of cardiac death, myocardial infarction, or the need for target-vessel revascularization within 9 months of implantation-occurred in $79(9 \%)$ and $89(11 \%)$ patients in the biolimus and sirolimus groups, respectively: thus, the novel stent was noninferior to the conventional device. Angiography, carried out in a subset of both groups, revealed no noteworthy differences between in-stent percentage diameter stenosis, and no aneurysms were detected in either group.The authors conclude that the biolimus-eluting stent offers an effective and safe alternative to the sirolimus-eluting stent; however, further studies are required to investigate long-term outcomes including the risk of very late stent thrombosis.

Original article Windecker S et al. (2008) Biolimus-eluting stent with biodegradable polymer versus sirolimus-eluting stent with durable polymer for coronary revascularisation (LEADERS): a randomised non-inferiority trial. Lancet 372: 1163-1173

\section{Patients with atrial fibrillation benefit from cardiac resynchronization therapy}

Cardiac resynchronization therapy (CRT) can reduce symptoms and improve cardiac function in patients who have congestive heart failure with cardiac dyssynchrony and left ventricular dysfunction; however, few data are available on the effects of CRT in patients with atrial fibrillation (AF) - this subgroup includes close to onethird of all patients with advanced heart failure. A meta-analysis by Upadhyay and colleagues has now shown that patients with AF experience notable improvements in symptoms and functional outcomes after CRT.

The authors identified four prospective cohort studies (and a substudy of a randomized clinical trial) that reported the effect of CRT on patients with AF and sinus rhythm in terms of mortality, left ventricular ejection fraction, and functional outcomes (determined by NYHA functional class, 6 min walk test, and quality of life measured by the Minnesota Living With Heart Failure ${ }^{\circledR}$ Questionnaire). Of the 1,164 patients (average age 66 years, $78 \%$ male) included in these five studies, 797 presented with sinus rhythm and 367 had AF.

The number of deaths after 1 year were similar (41 AF patients, 44 sinus rhythm patients, risk ratio $1.57, P=0.13$ ). Left ventricular ejection fraction improved significantly for both groups, although the change was slightly greater for patients with AF than for those with sinus rhythm. Functional benefits of CRT were slightly lower in patients with $\mathrm{AF}$ than in patients with sinus rhythm, but the overall benefit of $C R T$ in the patients with $A F$ remained significant.

Original article Upadhyay GA et al. (2008) Cardiac resynchronization in patients with atrial fibrillation: a meta-analysis of prospective cohort studies. J Am Coll Cardiol 52: 1239-1246 